\title{
Chirplet signal design by FPGA
}

\author{
Mohammed Jawad Al-Dujaili', Aws Majeed Al-Awadi² \\ ${ }^{1}$ Department of Electronic and Communication, Faculty of Engineering, University of Kufa, Iraq \\ ${ }^{2}$ Communication and Media Commission Baghdad, Iraq
}

\begin{tabular}{l}
\hline \hline Article Info \\
\hline Article history: \\
Received Dec 25, 2019 \\
Revised Sep 26, 2020 \\
Accepted Oct 7, 2020 \\
\hline
\end{tabular}

Keywords:

Chirp

Chirplet

FPGA

SAR

\begin{abstract}
The ever-expanding growth of the electronics and communications industries present new challenges for researchers. One of these challenges is the generation of the required bandwidth signal over a specific time frame that is used in a variety of contexts, particularly radar systems. To improve the range resolution in the radar along with better SNR, it is necessary to reduce the signal bandwidth and increase the peak power. There are some restrictions for narrowband signals like power limitation, pulse shaping, and the production of unwanted harmonics. So as a solution pulse compression techniques are suggested. Pulse compression is a process that modulating the transmitted pulse to achieve a wideband signal and then at the receiver, the received signal correlates with the transmitted pulse to achieve narrowband representations of data. Chirp is the most common signal used in pulse compression. The chirp signal is produced using linear frequency modulation. In this study, we attempted to add an amplitude modulation to the chirp signal and evaluate its performance by implementation on FPGA. The outcome signal is called chirplet and simulation will show that it enhance target detection and image quality in imaging radars like SAR.
\end{abstract}

This is an open access article under the $\underline{C C B Y-S A}$ license.

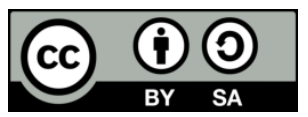

\section{Corresponding Author:}

Mohammed Jawad Al-Dujaili

Department of Electronic and Communication Engineering

Faculty of Engineering

University of Kufa, Iraq

Email: Mohammed.challab@uokufa.edu.iq

\section{INTRODUCTION}

Signal compression is an effective way to achieve the benefits of a short pulse and wideband signals at the same time. Attempts to produce a high bandwidth signal at the appropriate time, thus eliminating the need for very high power at short intervals as seen in short pulse signals [1-3]. One of the most widely uses of signal compression is in radar applications [4, 5]. Various implementation LFM methods have been studied in the articles [6-12]. The use of LFM modulation along with many features also has disadvantages. The LFM signal at the beginning and end of its spectrum has large amplitude jumps that cause signals to be added to the system in the frequency spectrum. Adding these frequencies disrupts the operation of the transmitter and receiver circuits, making it difficult to detect the reflected signal. In this paper, in order to overcome this problem, a domain modulation is also implemented on LFM modulation. Running the domain modulation softens the signal domain changes at the beginning and end of the LFM signal. The softening of the domain changes at the beginning and end of the spectrum has significantly increased the quality of the signal. The signal produced is called the Chirplet signal $[13,14]$. According to the widespread use of FPGA in the industry, this paper implements the chirplet signal using FPGA. Attempts have been made to use minimal FPGA resources, including LUT and FFT. This makes it possible to implement the system in lowcost hardware. The following section describes the importance of signal compression, and then in the third 
section, the chirplet signal will be explained. The fourth part of the paper describes the proposed algorithm and the fifth part shows the simulation results. Finally, the sixth section deals with the conclusion.

\section{IMPORTANT OF SIGNAL COMPRESSION IN RADARS}

To determine the target distance in the radar system, the delay time between the transmitted pulse and the echoed signal is calculated. For example, if the return pulse from the target has a delay time equal to $\tau$, the target distance from the radar will be $c \tau / 2$, where $\mathrm{c}$ is the speed of light [15-17]. Also, as seen in Figure 1, in order to distinguish return pulses from two adjacent targets, the transmitted pulse need to be sufficiently narrow. The range resolutions $(\Delta R)$ can be defined as $(1)[15,18,19]$ :

$$
\Delta R=\frac{c T_{p}}{2}
$$

where Tp is the width of the transmitted pulses. So the narrower the pulse gives a better resolution. On the other hand to achieve a better signal-to-noise ratio (SNR) it needed to Increase the signal peak power, which is difficult to achieve in practice by narrow pulse width [20-23].

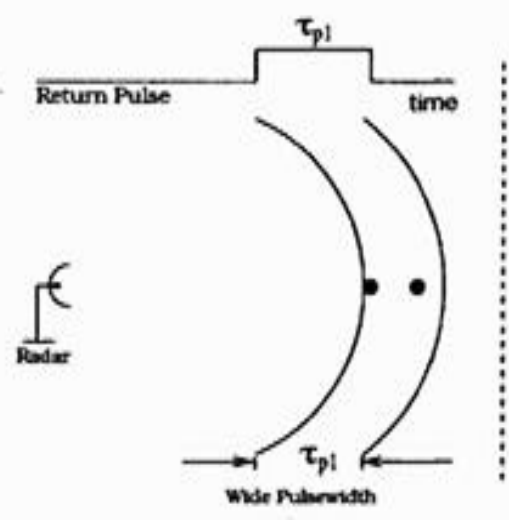

(a)

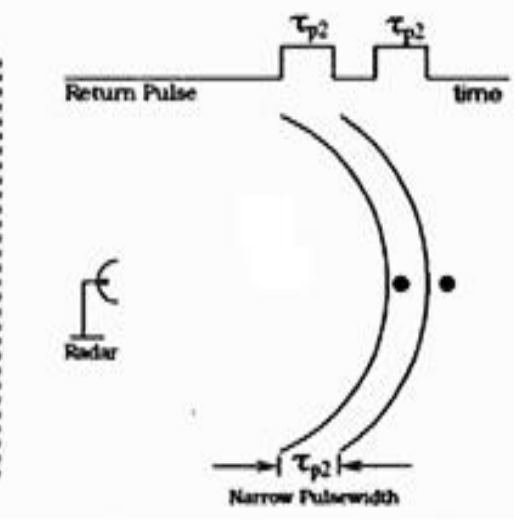

(b)

Figure 1. Radar system (a) targets can't be detected individually, (b) targets separated using narrow pulse width [18]

To fix this problem, wide bandwidth pulses are used. One of the most popular of these pulses is the linear FM modulation (LFM) pulse, known as the "chirp" signal Figure 2. LFM signal in terms of time is a relationship as (2).

$$
s(t)=P(t) e^{j \pi \alpha t^{2}} \quad-\frac{T_{p}}{2} \leq t \leq \frac{T_{p}}{2}
$$

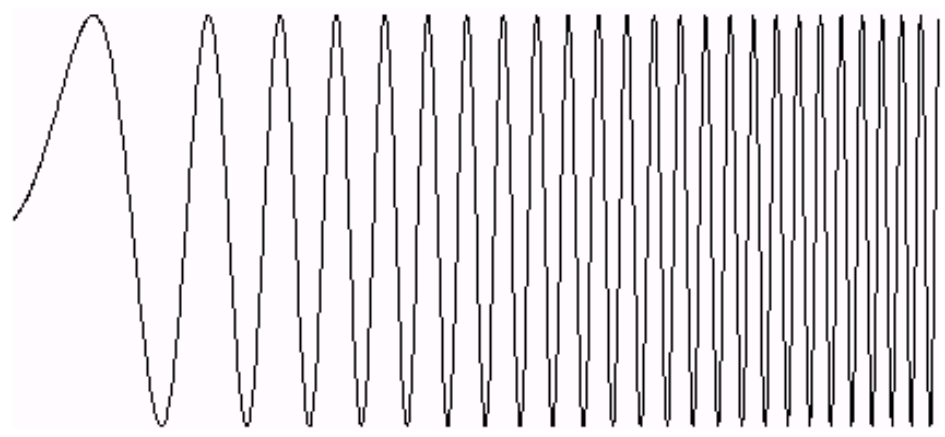

Figure 2. Chirp signal 
where $\mathrm{P}(\mathrm{t})$ is a square pulse at time $\mathrm{t}$, with the width of $\mathrm{Tp}$ and $\alpha$ is the rate of increase in frequency. In this signal, the instantaneous frequency increases linearly with time, so the wider pulse will have more bandwidth. The instantaneous frequency and bandwidth of this signal can be expressed by the parameters $f(t)$ and $\mathrm{B}$, as (3):

$$
f(t)=\frac{1}{2 \pi} \frac{d\left(\pi \alpha t^{2}\right)}{d t}=\alpha t, \quad B=\alpha T_{p}
$$

At the receiver, the pulse compression technique is used to separate targets $[24,25]$ as shown in Figure 3. This process consists of correlating between the received signal and a sample of sending chirp signals.

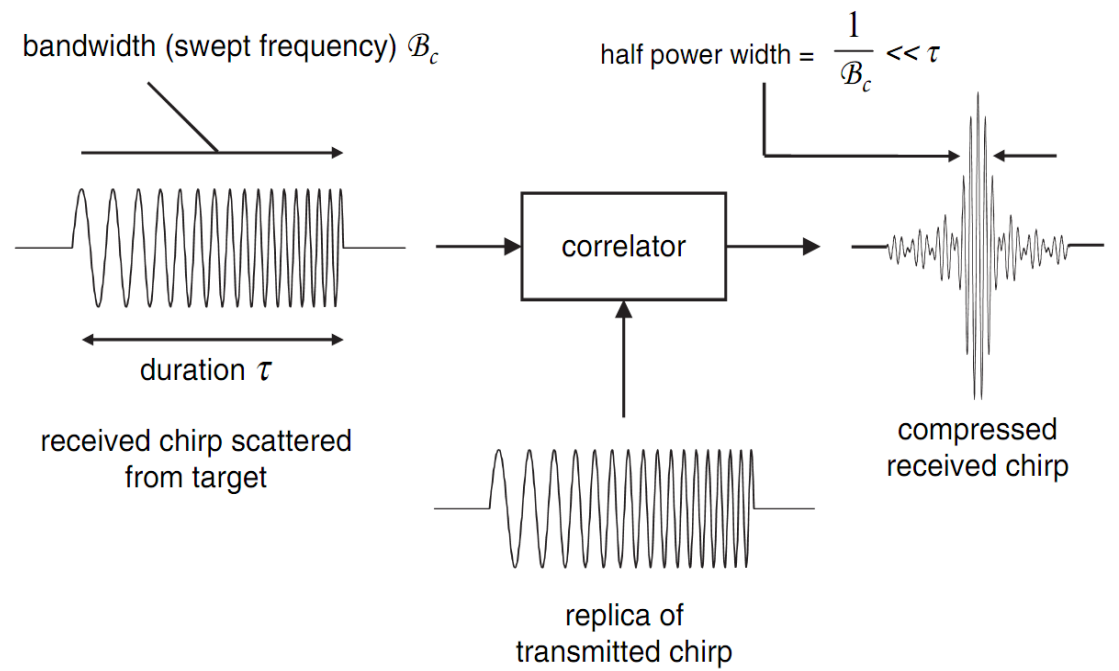

Figure 3. Chirp signal compression [24]

After compression, achieved resolution will be as (4).

$$
\Delta R=\frac{c}{2 B}
$$

In this case the resolution will be as (5).

$$
\Delta R=\frac{c}{2 B}=\frac{c}{2 \alpha T_{p}}
$$

So, unlike the narrowband pulses (1), the resolution becomes better as the pulse gets wider [26-32].

\section{CHIRPLET SIGNAL}

A chirplet is a windowed portion of a chirp signal, where the window provides some time localization property, like magnitude [33]. Signal amplitude variations can follow a variety of functions. For amplitude changes in terms of the Sine-Gaussian relation, the chirplet waveform is defined as the (6):

$$
S(t)=P(t) \exp \left(-\frac{B}{Q} t^{2}\right) \exp \left(j \pi \alpha t^{2}\right)-\frac{T_{p}}{2} \leq t \leq \frac{T_{p}}{2} \Delta R=\frac{c}{2 B}=\frac{c}{2 \alpha T_{p}}
$$

In the above relation $\mathrm{Q}$ is the signal quality coefficients (ratio of the central frequency to bandwidth). The waveform of a Chirplet signal is shown in Figure 4. The main advantage of using the Chirplet signal is the reduction of unwanted frequencies entered into the system. These frequencies cause by resistive nature of the signals against sudden changes in levels, like step increasing or decreasing. As a result, 
little distortions can be seen in both the primary and the terminal sides of the practical chirp signal. Chirplet omits these phenomena and achieves better impulse shape after the compression process for a point target. Therefore in imaging radars like synthetic aperture radar (SAR), higher quality images can be produced using chirplet [35-38].

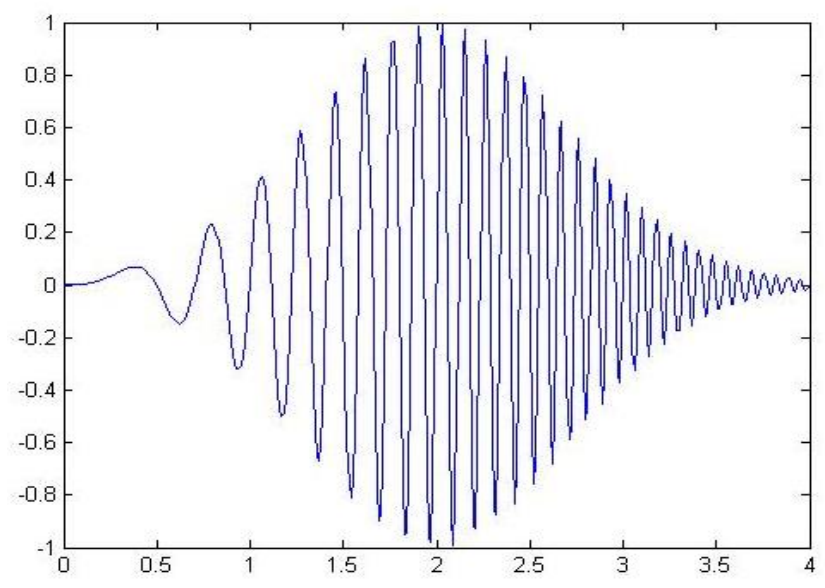

Figure 4. Sample of chirplet waveform [34]

\section{PROPOSED ALGORITHM}

In this section, the designed algorithm is fully described. In general, there are two ways to generate the desired signal, using LUT and direct digital signal generation. We will first describe each of these methods. LUTs are memories in which the values of the signal we want to generate are pre-stored, and in the FPGA, one of these values is sent to the output by each clock. The advantage of this method is its simplicity. In this way, we first generate the desired signal in different software programs such as MATLAB and sample its values. We then store these samples in the LUT. The disadvantage of this method is that in order to change the signal, the LUT must be re-programmed each time. Another way is to generate digital signals. In this method, we store only the values of a period of a sinusoidal signal in the LUT, and by selecting the number of LUT memory cells in specific iterations, frequency changes can be simulated. The difficulty of this method is the design of the address counter block. This block selects the values of phase differences according to the required frequency. The advantage of using this method over the previous method is the possibility of generating a signal with various parameters, and its drawback is more challenges in hardware design. Another advantage of this method is that to change the signal shape, we only need to adjust the small parameters and there is no need to design new values and load on LUT. In this article, the second method is used.

The first block designed to implement this research is scheduling control. Sending information to the transmitter is a repetitive operation with fixed time intervals. The number of pulses of a repeating signal in a specific time unit, normally measured in pulses per second is called the pulse repetition frequency (PRF). As a result, the FPGA requires a block design that can control the timing and repeat the transmitting at specified intervals. The perfect choice for this design is an Up-Counter. This block increases the number stored in the buffer per clock. This increment continues until the buffer reaches its maximum and then it resets to zero for the next round.

Chirplet signal design requires a chirp signal with appropriate amplitude changes. The chirp signal can be generated by controlling the phase changes of a sine wave. So at this point, we need two blocks. The first block controls the phase changes and the second block is the library that provides the sine value corresponding to the requested phase. This is because the FPGA does not have high-level commands such as geometric operations. The best way to implement these functions is to use the look-up tables (LUT). LUT is a memory in which the values sampled from a sinusoidal signal are stored. It is possible to generate the output signal with the required frequency by refreshing memory addresses at specified time intervals or by skipping the specified number of memory addresses. To reduce the stored data in memory, only half of the sin period is sampled and inserted into memory. This is because the other half of this signal is similar to the first half and only the sign is different. With the proper design of the phase controller, the LUT data once called with the positive sign and then with the negative sign. 
In the next block, when the enable signal is activated, the built-in up counter starts. The count range for this up-counter is between 0 and 1023. The coefficient of frequency increase in the written program is set to $25 \%$, which can be adjusted according to the radar chip rate.

The next step is to change the amplitude of the chirp signal and convert it to Chirplet. For this purpose, the Gaussian signal is used in this paper. As mentioned earlier, there is no high-level Gaussian signal function in the FPGA. As a result, the desired signal is generated by MATLAB and similar to the previous part the desired signal is generated.

To generate a Gaussian signal, the block receive an address between 0 and 1023 and then produces the appropriate amplitude. For address generation, a control block designed to control a rising counter. In the final step, the two generated signals must be combined. The mixer block is designed for this purpose. The chirp signal is 9-bit ( 8 bit+1 bit sign), and the Chirplet signal is 17 bit due to the multiplication of two 8-bit signals and the addition of one bit sign. The final output signals and block diagram of this design is as Figures 5 and 6.

\begin{tabular}{l|l}
$t \rightarrow$ out_me \\
$\rightarrow \rightarrow$ out_en
\end{tabular}

Figure 5. Final output signals

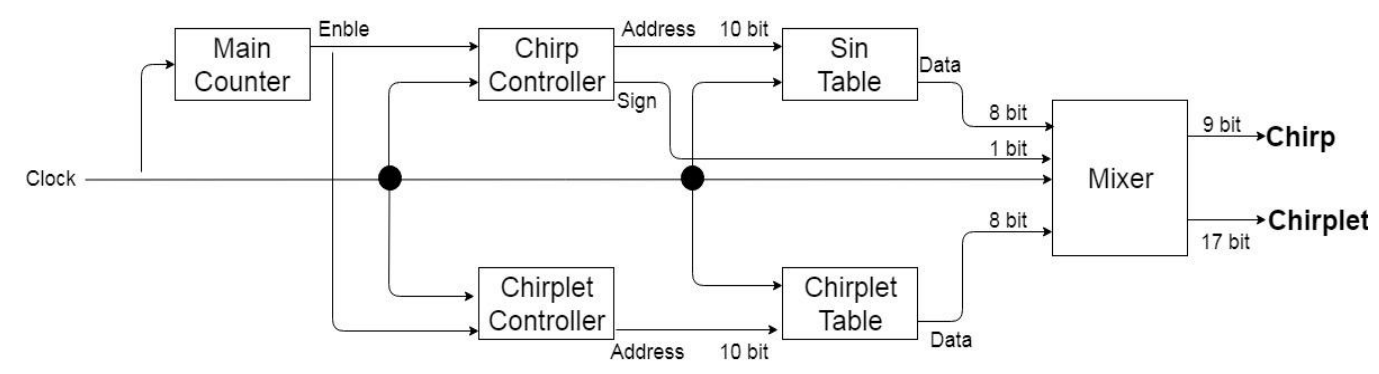

Figure 6. Overview of the relationship between blocks in the designed algorithm

\section{SIMULATION RESULTS}

MATLAB and ISE Xilinx software have been used for the simulation. In the first step, the resource consumption of FPGA was investigated in each method. The FPGA selected from the Spartan6 family with the specific type of xc6slx9. The obtained results can be seen in Table 1.

Table 1. Comparison of FPGA resources used in different methods

\begin{tabular}{cccc}
\hline Methods & LUT & FF & Delay \\
\hline [39] Chirp Signal & 94 & 91 & $2.54 \mathrm{~ns}$ \\
[40] Chirp Signal & 93 & 92 & $2.62 \mathrm{~ns}$ \\
[41] Chirp Signal & 102 & 85 & $2.84 \mathrm{~ns}$ \\
The proposed method & 116 & 104 & $3.15 \mathrm{~ns}$ \\
\hline
\end{tabular}

The reason for the increases in delay and greater uses of FPGA resources in the proposed method rather than in previous methods is the addition of amplitude modulation to the system. Due to the benefits of a designed system, such as better performance in signal detection and improved signal quality, these 
increments can be neglected. One of the evolution criteria in imaging radar algorithms is the integrated side lobe ratio (ISLR) and the peak side lobe ratio (PSLR). These measures indicate the degree of target concentration. This is done by calculating the energy of the sub lobe relative to the main lobe for a point target. To calculate the ISLR as in Figure 7(b), first an area of 5 blocks of resolution per dimension is selected to extract the energy of the main lobe, then as in Figure 7(c), this location filter is increased to 10 blocks. To determine the total energy of the main lobe and the side lobe. The ISLR value is then calculated using the (7).

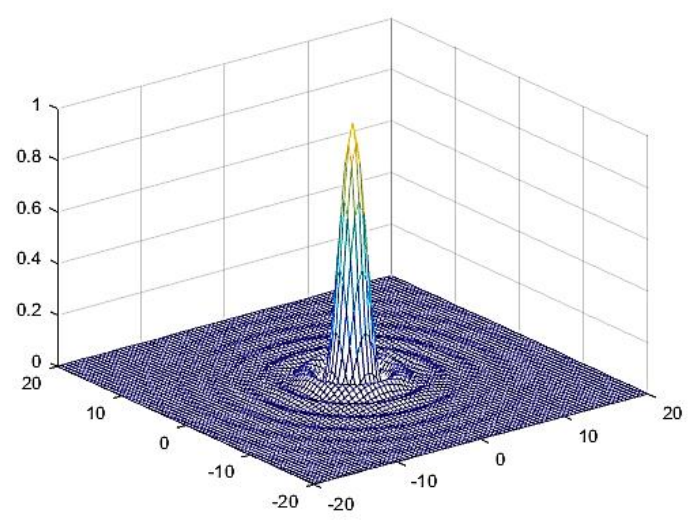

(a)

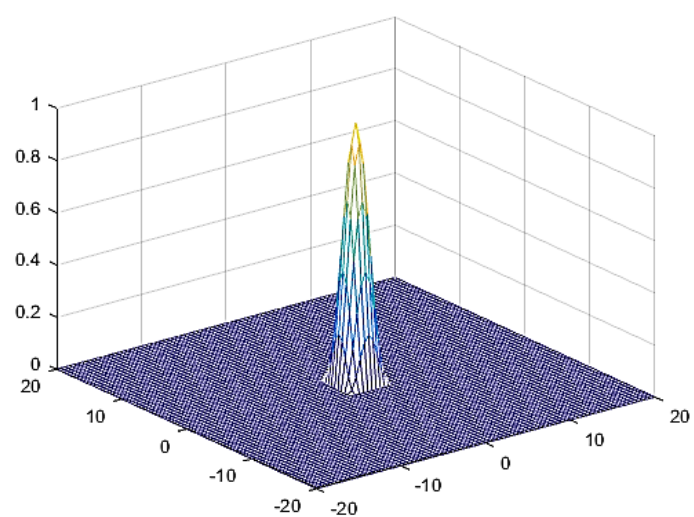

(b)

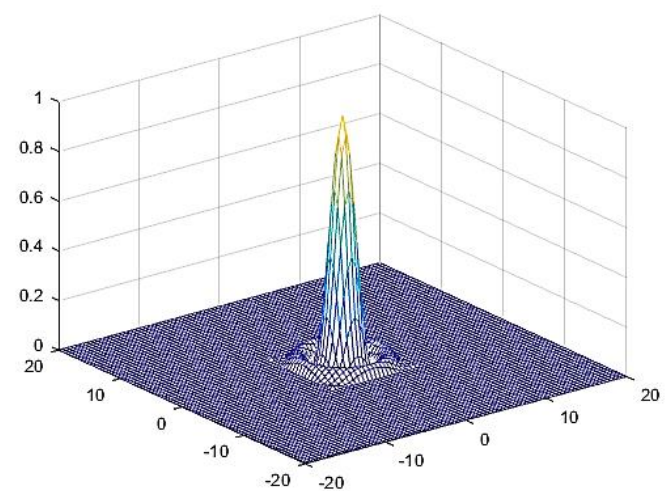

(c)

Figure 7. Point target (a) Signal from point target, (b) Main lobe, (c) Main lobe + sub lobe

$$
\left.I S L R\right|_{d b}=10 \log \left|\oiint_{(10 \Delta x .10 \Delta r)}\right| I\left|d A-\oiint_{(2.5 \Delta x .2 .5 \Delta r)}\right| I|d A|-10 \log \left|\oiint_{(2.5 \Delta x .2 .5 \Delta r)}\right| I|d A|(7)
$$

To calculate the PSLR maximum of the first side lobe divided by the maximum of the main lobe as seen as (8).

$$
\left.P S L R\right|_{d b}=190 \log \left|I_{S}\right|-10 \log \left|I_{M}\right|
$$

Given the values of Table 2 from the simulations, it is evident that chirplet output has better quality than a chirp signal.

Table 2. ISLR and PSLR values in different images

\begin{tabular}{cccc}
\hline & Chirp Signal [42] & Chirp Signal [39] & Chirplet Signal \\
\hline ISLR (db) & -7.19 & -10.31 & -7.5 \\
PSLR (db) & -13.82 & -13.62 & -14.1 \\
\hline
\end{tabular}




\section{CONCLUSION}

Signal compression is an important issue in telecommunications. Bandwidth signal generation and timing are of great importance in radar manufacturing. As mentioned earlier, the chirp signal is one of the methods of signal compression. Chirp signal, also has some disadvantage. The simulation results in this paper showed that the chirp signal in its frequency band has very disturbing frequencies that challenge the receiver with justification. In order to eliminate the disadvantages of the chirp signal, amplitude modulation was used on the chirp signal. The simulation results in this paper showed that the signal quality produced increased significantly. This paper deals with generating a chirplet signal in FPGA and shows the advantages of using a chirplet signal. The simulation results show that the chirplet signal uses more FPGA resources than the chirp signal due to more processing step, but contrast of radar image can Increases significantly

\section{REFERENCES}

[1] Y. Lee, V. Koo, and Y. Chan, "Design and development of FPGA-based FFT Co-processor for Synthetic Aperture Radar (SAR)," Progress in Electromagnetics Research Symposium-Fall (PIERS-FALL), 2017, pp. 1760-1766, doi: 10.1109/PIERS-FALL.2017.8293422.

[2] K. Anuraj and S. Poorna, "Performance Analysis of Optimization Algorithms Using Chirp Signal," International Conference on Inventive Computation Technologies, 2019, pp. 132-137.

[3] J. A. Finley and D. L. Woodell, "Radar system and method using pulse compression," ed: Google Patents, 2011.

[4] O. P. Pasquero, A. Hérique, and W. Kofman, "Oversampled pulse compression based on signal modeling: application to CONSERT/rosetta radar," IEEE Transactions on Geoscience and Remote Sensing, vol. 55, no. 4, pp. 2225-2238, 2017, doi: 10.1109/TGRS.2016.2639449.

[5] G. Franceschetti and R. Lanari, "Synthetic aperture radar processing," CRC press, 2018.

[6] S. Gong, X. Wei, X. Li, and Y. Ling, "Mathematic principle of active jamming against wideband LFM radar," Journal of systems engineering and electronics, vol. 26, no. 1, pp. 50-60, 2015, doi: 10.1109/JSEE.2015.00008.

[7] W. Fu, D. Jiang, Y. Su, R. Qian, and Y. Gao, "Implementation of wideband digital transmitting beamformer based on LFM waveforms," IET Signal Processing, vol. 11, no. 2, pp. 205-212, 2016, doi: 10.1049/iet-spr.2016.0114.

[8] V. N. Reddy, V. Lavanya, and B. Kiranmai, "Radar Pulse Compression Waveform Generation using FPGA Implementation," i-Manager's Journal on Digital Signal Processing, vol. 7, no. 2, p. 40, 2019, doi: 10.26634/jdp.7.2.16790.

[9] Y. Zhang, X. Chen, H. Xu, X.-P. Zhang, and F. Qi, "Fast Acceleration and Velocity Estimation for Wideband Stretching LFM Radars based on Mutual Bias Correction," IEEE Sensors Journal, vol. 20, no. 15, pp. 8683-8697, 2020, doi: 10.1109/JSEN.2020.2983839.

[10] T. Gu, G. Liao, Y. Li, Y. Guo, and Y. Huang, "Parameter estimate of multi-component LFM signals based on GAPCK," Digital Signal Processing, vol. 100, p. 102683, 2020, doi: 10.1016/j.dsp.2020.102683.

[11] Q. Guo, F. Zhang, P. Zhou, and S. Pan, "Dual-band LFM signal generation by optical frequency quadrupling and polarization multiplexing," IEEE Photonics Technology Letters, vol. 29, no. 16, pp. 1320-1323, 2017, doi: 10.1109/LPT.2017.2722004.

[12] Y. Wang, K. Wang, F. Jing, X. Lan, Y. Zou, and L. Wan, "LFM Signal Analysis Based on Improved Lv Distribution," IEEE Access, vol. 7, pp. 169038-169046, 2019, doi: 10.1109/ACCESS.2019.2954455.

[13] Y. Miao, H. Sun, and J. Qi, "Synchro-compensating chirplet transform," IEEE Signal Processing Letters, vol. 25, no. 9, pp. 1413-1417, 2018, doi: 10.1109/LSP.2018.2858018.

[14] F. Boßmann and J. Ma, "Asymmetric chirplet transform-Part 2: Phase, frequency, and chirp rateAsymmetric chirplet transform: Part 2," Geophysics, vol. 81, no. 6, pp. V425-V439, 2016, doi: 10.1190/geo2015-0696.1.

[15] B. R. Mahafza, "Introduction to radar analysis," Chapman and Hall/CRC, 2017.

[16] O. Boric-Lubecke, V. M. Lubecke, A. D. Droitcour, B.-K. Park, and A. Singh, "Doppler radar physiological sensing," Wiley Online Library, 2016.

[17] D. K. Barton, "Radar equations for modern radar," Artech House, 2013.

[18] C. G. Romero, "High Resolution Simulation of Synthetic Aperture Radar Imaging," digitalcommons.calpoly.edu, 2010.

[19] C. Alabaster, "Pulse doppler radar," The Institution of Engineering and Technology, 2012.

[20] G. Galati, G. Pavan, and F. De Palo, "Chirp signals and noisy waveforms for solid-state surveillance radars," Aerospace, vol. 4, no. 1, p. 15, 2017, doi: 10.3390/aerospace4010015.

[21] A. De Maio, Y. C. Eldar, and A. M. Haimovich, "Compressed Sensing in Radar Signal Processing," Cambridge University Press, 2019.

[22] J. P. Fitch, "Synthetic aperture radar," Springer Science \& Business Media, 2012.

[23] L. Anitori, A. Maleki, M. Otten, R. G. Baraniuk, and P. Hoogeboom, "Design and analysis of compressed sensing radar detectors," IEEE Transactions on Signal Processing, vol. 61, no. 4, pp. 813-827, 2012, doi: 10.1109/TSP.2012.2225057.

[24] W. G. Carrara, "Soptlight synthetic aperture radar," Signal Processing Algorithms, 1995.

[25] I. Intyas, R. Hasanah, M. R. Hidayat, B. Hasanah, A. B. Suskmono, and A. Munir, "Improvement of radar performance using LFM pulse compression technique," International Conference on Electrical Engineering and Informatics (ICEEI), 2015, pp. 302-307, doi: 10.1109/ICEEI.2015.7352515. 
[26] A. G. Voronovich and V. U. Zavorotny, "Bistatic radar equation for signals of opportunity revisited," IEEE Transactions on Geoscience and Remote Sensing, vol. 56, no. 4, pp. 1959-1968, 2017, doi: 10.1109/TGRS.2017.2771253.

[27] H. D. Griffiths and C. J. Baker, "An introduction to passive radar," Artech House, 2017.

[28] B. R. Mahafza, "Radar signal analysis and processing using MATLAB," CRC Press, 2016.

[29] A. Aubry, A. De Maio, Y. Huang, M. Piezzo, and A. Farina, "A new radar waveform design algorithm with improved feasibility for spectral coexistence," IEEE Transactions on Aerospace and Electronic Systems, vol. 51, no. 2, pp. 1029-1038, 2015, doi: 10.1109/TAES.2014.140093.

[30] H. You, X. Jianjuan, and G. Xin, "Radar data processing with applications," John Wiley \& Sons, 2016.

[31] M. C. Budge and S. R. German, "Basic radar analysis," Artech House, 2015.

[32] R. M. Rauber and S. L. Nesbitt, "Radar Meteorology: A First Course," John Wiley \& Sons, 2018.

[33] Y. Lu, E. Oruklu, and J. Saniie, "Chirplet signal and empirical mode decompositions of ultrasonic signals for echo detection and estimation," Journal of Signal and Information Processing, vol. 4, no. 02, p. 149, 2013, doi: 10.4236/jsip.2013.42022.

[34] S. Mohapatra, Z. Nemtzow, É. Chassande-Mottin, and L. Cadonati, "Performance of a chirplet-based analysis for gravitational-waves from binary black-hole mergers," Journal of Physics: Conference Series, vol. 363, no. 1, pp. 1-7, 2012.

[35] Y. Wang, Z. Wang, B. Zhao, and L. Xu, "Compensation for high-frequency vibration of platform in SAR imaging based on adaptive chirplet decomposition," IEEE Geoscience and Remote Sensing Letters, vol. 13, no. 6, pp. 792-795, 2016, doi: 10.1109/LGRS.2016.2544945.

[36] G. Jin, X. Gao, X. Li, and Y.-g. CHEN, "A high velocity motion compensation algorithm of ISAR echo based on chirplet transform," Journal of Astronautics, vol. 31, no. 7, pp. 1844-1849, 2010.

[37] G. Yu and Y. Zhou, "General linear chirplet transform," Mechanical Systems and Signal Processing, vol. 70, pp. 958-973, 2016, doi: 10.1016/j.ymssp.2015.09.004.

[38] Y. Wang and Y. Jiang, "ISAR imaging for three-dimensional rotation targets based on adaptive Chirplet decomposition," Multidimensional Systems and Signal Processing, vol. 21, no. 1, p. 59, 2010.

[39] H. Yang, S.-B. Ryu, H.-C. Lee, S.-G. Lee, S.-S. Yong, and J.-H. Kim, "Implementation of DDS chirp signal generator on FPGA," International Conference on Information and Communication Technology Convergence (ICTC), 2014, pp. 956-959, doi: 10.1109/ICTC.2014.6983343.

[40] R. Spoorthi "FPGA Implementation of Linear Frequency Modulation (LFM) Waveforms for Radar," Journal of Digital Integrated Circuits in Electrical Devices, vol. 2, 2017.

[41] B. D. Putera, E. Ali, H. Wijanto, and M. Ramdhani, "Design and Realization of Memory-Based Chirp Generator on Synthetic Aperture Radar (SAR)," Journal of Measurements, Electronics, Communications, and Systems, vol. 4, no. 1, pp. 16-19, 2018, doi: 10.25124/jmecs.v4i1.1695.

[42] S. K. Das, "Synthetic aperture radar image quality measurements," diva-portal.org, 2010.

\section{BIOGRAPHIES OF AUTHORS}

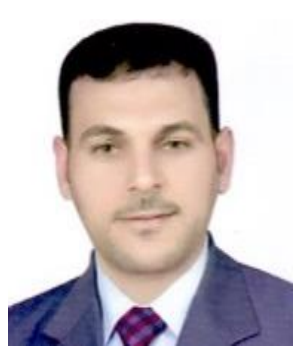

Mohammed Jawad Al-Dujaili Al-Khazraji awarded B.S. degree in communication engineering from University of Al-Furat Al-Awsat Technical, Technical College of Engineering, Najaf, Iraq in 2008 and M.S. degree in communication system engineering from Ferdowsi university, Iran, in 2018. Currently, he is a member staff at the Department of Electronic and Communication, Faculty of Engineering, University of Kufa, Iraq. His research interest includes the development of Wireless communications and signal processing as well as image, speech processing and radar, 5G. Email: Mohammed.challab@uokufa.edu.iq

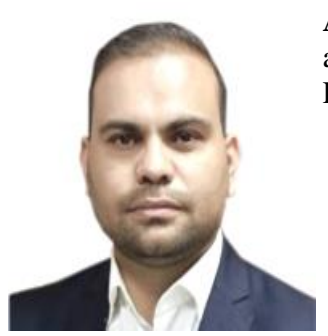

Aws Majeed Al-Awadi Associate Director of the communication Regulatory Authority, Head of allocation frequency affairs Department Communication and Media Commission, Baghdad, Iraq. Email: A.majeed@cmc.iq 